BULLETIN OF THE

AMERICAN MATHEMATICAL SOCIETY

Volume 77, Number 2, March 1971

\title{
FIXED POINT SCHEMES
}

\author{
BY JOHN FOGARTY ${ }^{1}$
}

Communicated by Murray Gerstenhaber, October 26, 1970

Let $S$ be a scheme and let $G$ be a group scheme over $S$. If $\alpha: G \times X$ $\rightarrow X$ is an action of $G$ on $X$ over $S$ (cf. [4]), we say that $(X, \alpha)$-or simply $X$-is a $G$-scheme over $S$. The 'fixed point functor' $h_{X}^{G}$ of $G$ in $X$ is defined as follows. For each $S$-scheme $Y$, let $Y_{G}$ denote the trivial $G$-scheme $\left(Y, p_{2}\right)$. Then

$$
h_{X}^{G}(Y)=\left(\text { set of } G \text {-linear } S \text {-morphisms } \varphi: Y_{G} \rightarrow X\right) .
$$

Theorem 1. If $\mathfrak{C}$ is the category of locally noetherian S-schemes and quasicompact $S$-morphisms, $X$ is a $G$-scheme in $\mathfrak{C}$, and $G$ is flat over $S$, then $h_{X}^{G}$ is represented by a closed subscheme $X^{G}$ of $X$.

In this vast generality it is not to be expected that much detailed information about $X^{a}$ can be obtained. Nevertheless, one does have the following 'rigidity' result when $G$ is an abelian scheme over $S$ (cf. [4]).

TheOREM 2. Let $G$ be an abelian scheme over $S$ and let $X$ be a connected locally noetherian $G$-scheme over $S$. Then either $X^{G}$ is empty or $X^{G}=X$.

It is conceivable that this property could be used as the starting point for the general theory of abelian schemes, e.g., commutativity and Chow's theorem (cf. [3]) are easy consequences of Theorem 2.

For a deeper study of fixed point schemes, we restrict ourselves to the category of algebraic schemes over a field $k$, acted upon by algebraic groups (i.e., smooth group schemes of finite type) over $k$. One result, which is related to a special case of a recent result of $G$. Horrocks [2], is

Proposition 3. Let $G$ be a linear algebraic group over $k$. The largest $k$-closed normal subgroup $H$ of $G$ such that, for all proper connected $G$-schemes $X$ over $k, X^{H}$ is connected is the unipotent radical of $G$.

For smooth schemes and 'very good groups' one has:

AMS 1970 subject classifications. Primary 14L15.

Key words and phrases. Group scheme, action, fixed point, abelian scheme, unipotent group, linearly reductive group.

1 The author was supported by a grant under NSF-GP-25329. 
PROPOSITION 4. If a linearly reductive linear algebraic group $G$ acts on a smooth algebraic scheme $X$ over $k$, then $X^{G}$ is smooth over $k$.

It seems to be an open question whether $X^{G}$ is smooth in the case of a semisimple group $G$ acting on a smooth $X$ over a field $k$ of characteristic $p>0$. This is false for finite groups $G$ such that $p$ divides the order of $G$.

\section{REFERENCES}

1. J. Fogarty, Fixed point schemes, Amer. J. Math. (to appear).

2. G. Horrocks, Fixed point schemes of additive group schemes, Topology 8 (1969), 233-242. MR 39 \#5578.

3. S. Lang, Abelian varieties, Interscience Tracts in Pure and Appl. Math., no. 7, Interscience, New York, 1959. MR 21 \#4959.

4. D. Mumford, Geometric invariant theory, Ergebnisse der Mathematik und ihrer Grenzgebiete, Band 34, Academic Press, New York; Springer-Verlag, Berlin and New York, 1965. MR 35 \#5451.

University of Pennsylvania, Philadelphia, Pennsylvania 19104 